\title{
Improving reproducibility and external validity. The role of standardization and data reporting of laboratory rat husbandry and housing ${ }^{1}$
}

\author{
José Luiz Fontoura-Andrade', Rivadávio Fernandes Batista de Amorim", João Batista de Sousa'"I
}

'Fellow Master degree, Postgraduate Program in Medical Sciences, School of Medicine, Universidade de Brasilia (UnB). DVM, Experimental and Compared Surgery Laboratory, Armed Forces Hospital, Brasilia-DF, Brazil. Acquisition of data, manuscript writing.

"Ph D, Associate Professor, Department of Pathology, School of Medicine, UnB, Brasilia-DF, Brazil. Manuscript writing, critical revision.

IIIPhD, Associate Professor, Department of Surgery, School of Medicine, UnB, Brasilia-DF, Brazil. Manuscript writing, critical revision.

\section{Abstract}

Purpose: To identify the most relevant flaws in standardization in husbandry practices and lack of transparency to report them. This review proposes some measures in order to improve transparency, reproducibility and eventually external validity in experimental surgery experiments with rat model.

Methods: We performed a search of scientific articles in PUBMED data base. The survey was conducted from august 2016 to January 2017. The keywords used were "reproducibility", "external validity", "rat model", "rat husbandry", "rat housing", and the time frame was up to January 2017. Articles discarded were the ones which the abstract or the key words did not imply that the authors would discuss any relationship of husbandry and housing with the reproducibility and transparency of reporting animal experiment. Reviews and papers that discussed specifically reproducibility and data reporting transparency were laboriously explored, including references for other articles that could fulfil the inclusion criteria. A total of 246 articles were initially found but only 44 were selected.

Results: Lack of transparency is the rule and not the exception when reporting results with rat model. This results in poor reproducibility and low external validity with the consequence of considerable loss of time and financial resources. There are still much to be done to improve compliance and adherence of researchers, editors and reviewers to adopt guidelines to mitigate some of the challenges that can impair reproducibility and external validity.

Conclusions: Authors and reviewers should avoid pitfalls of absent, insufficient or inaccurate description of relevant information the rat model used. This information should be correctly published or reported on another source easily available for readers. Environmental conditions are well known by laboratory animal personnel and are well controlled in housing facilities, but usually neglected in experimental laboratories when the rat model is a novelty for the researcher.

Key words: Reproducibility of Results. Models, Animal. Rats. 


\section{- Introduction}

Translational research is the multidirectional flow of data between different levels of basic, preclinical and clinical studies. Experimental surgery plays a relevant role in translational research because it is capable to provide specific preliminary information as preclinical study or supplementary data along ongoing clinical studies. Low reproducibility is the inability to replicate the majority of findings in animal experiments presented in journals ${ }^{1}$. External validity is the applicability of the results to clinical practice, it is the extent that the results of a study can be generalized as reliable data from the study population (rat model) to preclinical and clinical studies. Reproducibility and external validity are different concepts but are interrelated when thinking about reliable translational research.

Expenses with preclinical research which results are not reproducible or have serious problems with external validity are estimated to cost 28 billion dollars per year only in the USA ${ }^{2}$. Low reproducibility and poor external validity can be caused by several factors, such as: inadequacy of animal model, poor experiment design, inaccurate statistical analysis ${ }^{3}$, environmental conditions and even publication bias.

International Committee of Medical Journal Editors advises that the methods section of an article must be sufficiently detailed so that other researchers who have access to data could be able to reproduce experiment and results. In such scenario, the guideline known as ARRIVE (Animal Research Reporting In Vivo Experiments) has been proposed to assist authors, reviewers and editors involved in animal experimentation publications4. The ARRIVE has been adapted and used by journals in different fields (i.e. Pathology, behavioural neurosciences). However, its recommendations have not been properly encouraged by the editors ${ }^{5}$ and it is very rare to retrieve a paper that all ARRIVE recommendations are fully and adequately described.

\section{Selection of the appropriate rat model}

Researchers must question themselves if rat model is adequate for the specific purpose of the research experimental surgery or whether another laboratory animal or surrogate methods are options capable of more reliable results for external validity. This a very important ethical as well as a practical issue, certain areas of research still persist to adopt rat as model, despite poor achievements in translational results for human application. The vast majority of drugs that had have success in animal model and specifically in rat model fails when tested in human trials. Rat model must be justified and validated by scientific evidences. One form of validation is to compare preliminary results from animal studies with their subsequent clinical research in humans. Rat model can be adequate for some lines of research in experimental surgery, but there are serious limitations for modelling comorbidities like obesity, diabetes, hypertension, cardiac insufficient. Usually, young health rats are used as models. This limitation can be ameliorated with the choice of a suitable rat strain to model comorbidities related to a specific experimental surgery.

The most adequate strain should be extensively investigated before the onset of the experimental phase to prevent bias. One option is to design the experiment in randomized blocks with different strains ${ }^{6}$. If one or more comorbidities are relevant in clinical situation, they should be included in the design of the experiment. It is important to mention that there are strains or transgenic modified rat designed for several diseases and 
conditions, such as cardiovascular disease; alcohol-related behaviour, alcohol abuse, drug addiction, colon cancer, osteoarthritis, pain perception and conditioned fear, body fat distribution, epilepsy, hypertension, diabetic neuropathic pain, Parkinson's disease, spondyloarthropathies and etc. Hence, it must be taken into account available strains in the institution that the research will take place in order to achieve most consistent and reliable results. For instance, there are at least two strains that could be used in modelling human colon cancer. Both the polyposis in the rat colon (Pirc) and Kyoto Apc Delta (KAD) strains carry mutations in the intestinal-cancer-associated adenomatous polyposis coli (Apc) gene?

One good example to corroborate the importance of a judicious selection of rat strain is the right rat strain model for atherosclerosis. Only genetically altered strains mimic models of obesity, hyperlipidaemia or nephropathy, and only some altered strains are susceptible to fast atherosclerosis induced by a diet enriched with cholesterol. Although the majority of experimental atherosclerosis studies uses an outbred rat wild-type strain, mainly the Sprague-Dawley or Wistar rat. Both strains are inadequate models because they do not express plasma cholesteryl ester transfer protein and are resistant to cardiovascular diseases ${ }^{8}$.

\section{- Age of the rat}

Many studies use an arbitrary age (usually young animals) without considering possible differences between immature and mature subjects. In a recent review on rodent models for obesity, 4 papers in 19 (21.05\%) did not report the age of the subjects used and $13 / 19$ (68.42\%) of the studies adopted immature animals younger than 8 weeks of age ${ }^{9}$. A reliable method to compare human and rat age is yet to be determined. Physiological events can be used as indicators of onset and ending of specific life periods like the period between birth and weaning which could be compared to neonatal period of humans, after weaning and before sexual maturity to model human infancy, soon after epiphyseal growth plate fusion in tibiae as young mature adults, between sexual maturity and sexual senescence as model to mature humans, post sexual senescence period to model senile humans10. Nonetheless, these events vary according to strain and specific information must be investigated before setting the experiment. For instance, modelling postmenopausal osteoporosis in rats can be achieved by chemical manipulation or ovariectomy. Usually, juvenile rats are chosen for this kind of intervention in experiments. The question whether these perimenopause models are adequate if used in young rats persists, presumably, mature rats in post senescence period would mimic better conditions of a human female in perimenopause. Life's period is an important issue and a consistent safety margin between two patent physiological events must be considered. Reductionism or simplistic calculation factor must be avoided to correlate human and rat age.

\section{Choice of sex}

Males rats have been extensively used in most studies ${ }^{11}$. The prejudice against using female rats may be partly due to the assumption that they are intrinsically more variable than males because of cyclical reproductive hormones. Human females are more susceptible than males in diseases like: anxiety, depression, stroke, multiple sclerosis and obesity. In some areas of research, there are significant difference between genders and this is an important justification for investigate both sexes.

The current male bias in 8 out of 10 biological disciplines tends to decline ${ }^{11}$. The proposal of single-sex studies for candidates 
for research should be exception and not the rule and should only be accepted with robust justification. Before experimental design is proposed, researchers should conduct a literature review to learn known, unknown or inconclusive sex differences in the research area $^{12}$.

Learning influence of sex at molecular, cellular, biochemical levels and complex process like inflammation and cicatrization only will be achieved when studying both sexes become a guiding principle in biomedicine. For this reason, studies should incorporate females and males in equal numbers with explicit comparison of both sexes ${ }^{13,14}$.

\section{- Sanitary condition and intestinal microbiota}

There is no universal consensus for the assessment of pathogen status in rodent populations neither internationally standards criteria of their quality. Therefore, external validity and reproducibility can be impacted. On the other hand, there are some recommendations towards health monitoring for rodents by the Federation of European Laboratory Science Associations. An initiative to standardize the reporting of health status information for international transfer of rodents has been proposed as well ${ }^{15}$.

Interindividual variation in gut microbiota composition has been implicated as major cause of interindividual variation in systemic disease parameters and should not be ignored. Unfortunately, the current knowledge is not sufficient to clearly define whether any research in experimental surgery is gut microbiota-sensitive or not. The information about gut microbiota characterization is gradually increasing and microbial surveillance is expected to be a critical part of the animal model description ${ }^{16}$. If transgenic or genetic rodent models are purchased separately of the controls, cohousing all animals during acclimatization period is one form to prevent effects related to microbiota. In addition, several factors can influence microbiota such as mother of the litter, commercial source, husbandry conditions of the facility, cage system, diet composition and antimicrobial treatment (autoclave or irradiation of the diet), vaginal versus caesarean delivery, psychological stress, housing density, time of sample collection, type of bedding, frequency of bedding change and water quality. While it is not feasible to investigate and register gut microbiota composition for all published animal-based research, factors known or suspected to modulate should be reported whenever possible.

\section{- Dietary composition, weight and feeding}

One of the most frequent incomplete information regarding the rat husbandry is the description of animal feeding as "standard rat chow and water were provided ad libitum". This inaccurate description can jeopardize the reproducibility and external validity considering that different feedings can influence the results ${ }^{17}$. The food block brand name, manufacturer and the description of water quality should be reported on the paper. For long term studies, maybe some kind of calorie restriction should be implemented to avoid obesity and insulin intolerance ${ }^{18}$. There are several differences in physiological values for rats fed either ad libitum or by a caloric restriction diet, listed as follows: body weight, total body fat, mean blood pressure, resting heart rate, plasma glucose, plasma insulin, plasma leptin, plasma adiponectin, total cholesterol, low density lipoprotein; high density lipoprotein, TNF $\alpha$, and IL-6 levels. Special diets can be designed to mimic human disease co-morbidities like human metabolic syndrome, obesity and diabetes. One example of special diet is the cafeteria diet, which uses grocery store-purchased food items such as cookies, chips, and processed meats with 
high-fat, high-sugar, high-salt, and low-fiber ${ }^{19}$. Frequently, laboratory's rats are overweighed ad libitum-fed rodents, sedentary, obese and glucose intolerant. Ad libitum fed rat exhibits elevated levels of glucose, insulin, triglycerides, low-density lipoprotein (LDL) cholesterol, leptin, mean blood pressure, resting heart rate, TNF $\alpha$, IL-6 and decreased levels of adiponectin and ghrelin ${ }^{18}$ compared to moderately fed lean animals. Total body fat should provide a more accurate criterion for rat selection instead of weight alone and it can be up to $40 \%$. Without diet restriction, there is a moderate positive correlation of age and total body fat (i.e. younger animals show less total fat than older). Very young rats do not have time to accumulate fat despite of feeding. The distribution of body fat varies significantly between rat strains, diet composition, caloric restriction, exercise and all of these factors should be considered to design the right model for the experiment ${ }^{20}$. Rats with access to voluntary exercise in wheel running had less accumulation of fat than the sedentary ones ${ }^{21}$. Exercise can be also related to environmental enrichment.

\section{Environmental enrichment}

Laboratory rats are bred and kept in small, minimalist and monotonous standard cages. The development and responses of animals under these conditions are often unrepresentative and idiosyncratic. As a result, originated data are likely to have reduced external validity especially in research areas involving any behaviour assessment. Standard laboratory housing may result in low levels of cognitive function or altered levels of anxiety and this interference can jeopardize study of genetic and environmental risk factors for neuropsychiatric disorders ${ }^{22}$. In experimental surgery, published data reports that environmental enrichment had beneficial effects on brain trauma rat models, spinal cord injury and experimental stroke and mitigated the adverse effects of anaesthesia and surgery on postoperative cognitive function and on the development of neuroinflammation in a rat abdominal surgery model ${ }^{23,24}$.

A myriad of physical objects can be used as environmental enrichment elements as follows: increased surface area (larger cage) and/or inclusion of platforms to the cage or a 2-level cage, use of shelters for hiding places, nest lets, exercises, running wheels, craw ball, wood ball, plastic commercially available toys, bells/chimes, wooden chews and toys, swings, ropes, chains, ladders, ramps, pipes/ tubes/tunnels, foraging nesting material, shredded paper, rearrangement of food and water supply.

Environmental enrichment is an experimental variable, therefore it requires standardization and proper description to improve reproducibility of the experiment ${ }^{24}$. During design phase of the experiment, the researcher should review the possibilities of enrichment and choose the adequate ones, taking on account the field of research, size of the enriched cages, density, rat strain, rat gender, age at onset of enrichment, duration of enrichment, appropriate control group. The objects as well as the number supplied, frequency of provision and renewal should be registered and described.

\section{Acclimatization and habituation to experimental conditions}

Acclimatization is a period granted for the animal to return to physiological parameters after transportation. It provides the opportunity for adaptation to new surroundings and handlers. Even with all the measures taken in a well-planned and conducted transportation by commercial vendors, animals are submitted to a sort of different noises, odours, vibrations and light.

Acclimation period can vary significantly, depending on which physiological 
parameters were adopted to monitor.

The light-dark cycle is usually modified as light cycles cannot be controlled in transport vans or in other methods of transportation and at the arrival in the research facility. If no information is found while designing a study, the researcher should diligently pursue the establishment of a reasonable acclimatization period in a pilot study. Finally, complex evaluations like cognitive abilities and circadian circles can demand more time to return to pre transportation levels.

Even transportation of any duration in facility indoors can potentially affect rats and experiment results.

Habituation period before any experiment is mandatory; the time should be decided and rigorously followed. It is important to allow a standard time for the rat in the surgery room for the physiological parameters to return to previous levels, this is important for the safety of the subject in induction of anaesthesia and during anaesthesia itself. Habituation period after the experiment depends on the field of the research, it is relevant for behavioural and cognitive studies. In experimental surgery, it is more related to practical issues related to the return of an awaken subject to an individual cage or if possible, to its original cage and group.

\section{Interaction between humans and rats}

Fear and stressful situations related to human contact can significantly influence the test results. The habituation to handling is simple nevertheless is time consuming and requires investment in education of caretakers and researchers. Some programmes to minimize time and training with standard procedures and still produce results have been proposed. Individual gentling, which involves interaction with rats by touch, voice and food reward for some minutes every day beside minimizing fear of human contact, also increases the stability of the rat's reactions ${ }^{25}$. Human-rat interaction may be a positive adjunct to conventional behavioural training techniques and a possible confounder in noncontrolled experiments. A step beyond gentle handling habituation is playful handling or tickling, it reliably evoked emission of $50-\mathrm{kHz}$ ultrasonic, increased ease of handling and reduced anxiety-related responses in standard behaviour tests compared with minimal handling or passive hand exposure ${ }^{26}$. College of Veterinary Medicine of Purdue University explains and shows how to tickle rats in its website http://vet.purdue.edu/discovery/ gaskill/resources.php. Manipulation of rats in experimental surgery is needed in critical situations as just before the anaesthesia and immediate postoperative care.

\section{- Monitoring and informing light management}

In experimental surgery the importance of influence of light intensity variations that can be found inside transparent plastic cages on racks or shelves, depending on their positioning relative to the light source $^{27}$ and could lead to bias but it is not as important as in other research lines like behaviour and oncology in which rats are kept for extensive periods of time. Nevertheless, intense light employed for routine procedures in the surgery room stresses the rat and can irreversibly affect the retina ${ }^{28}$. The rat when in the surgery room should be protected from the intense light by covering the cage, and by covering its eyes during surgery, especially if dissociative anaesthetic agents are employed.

Many published animal studies do not inform the wavelengths neither of the light phase nor the dark phase. It is common practice to use red lamps in housing areas during dark phase to allow staff to visualize cages and animals without disturbing them. In experimental surgery, all the preanesthetic 
procedures can be conducted in a dark room with a small red light which should be verified if it produces wavelenghs greater than $640 \mathrm{~nm}$ and less than 15 lux. Any light source produces bands of wavelengths in the visible region of the electromagnetic spectrum (360 to 770 nanometers). The spectral power distribution is a pictorial representation of these bands. If the laboratory is not equipped to measure spectral power distribution, light source for both phases should be appropriately referenced. This information can be obtained as long as it is usual to lamp manufacturers to publish spectral power distribution curves of specific light sources ${ }^{29}$.

Most papers inform the length of time for dark and clarity (photoperiod), but usually an important information is not reported: the exact hour for turning the lights on. This will inform whether the cycles are synchronized with daytime or not. The laboratory rat has nocturnal habits; it rests during light time and is active in dark. If the light in the housing is kept on during the daytime, when the staff is active, handling the rats, conducting the experiments or even the noise produced by routine tasks, it can impact the resting period of the animal. One paper was very specific about the time of a behavioural experiment: in the first hour of the dark phase of the light/ dark cycle $^{30}$. Laboratory rats retain some vestiges of photoperiodic responsiveness ${ }^{31}$ and there are minor differences in susceptibility to influences of photoperiod between strains of laboratory rats $^{32}$. Heideman et al. ${ }^{33}$ defined that photoperiods $\leq 12.5$ hours fully suppressed reproduction and somatic growth the critical photoperiod of isogenic strain of Rattus norvegicus Fischer 344. This demonstrates that 12 hour photoperiod which is standard in some laboratory colonies has significant effects on reproductive maturation and growth rate of this rat strain. Researchers should check literature if the standard photoperiod of 12 hours of light and 12 hours of dark is suitable for their specific experiment.

\section{Social housing}

It is well documented that social isolation is harmful for rats. Isolation stress alters physiological and behavioural characteristics $^{34}$. On the other hand, more consistent results are achieved if animals are grouped in familiar and stable groups ${ }^{35}$. Most recommendations follow a relation between cage densities versus individual animal weight. In long term experiments, the final estimate weight should be calculated in order to group animals accordingly to final cage density. Therefore, the researcher will keep the same group from the beginning to end of experiment and will avoid separation of animals for cage density adjustments. Regrouping animals can be an uncontrolled variable and should be avoided.

\section{- Noise exposure levels}

It is impossible to perform routine husbandry activities in rats room, laboratory and surgery room without creating any sound. The intensity and duration of sound pollution produced during these activities can vary between individual animal caretakers. If no standardization is imposed, great differences between styles of working of the animal care personnel can occur. Efforts should be done to educate animal caretakers in order to minimize sound disturbances with appropriate technique in all regular activities, like pouring diet into food hoppers, cleaning, changing cage grids and water bottles. Rat sleep is easily disturbed by environmental noise exposure, thus, acoustic environment of laboratory facilities must avoid excessive high-frequency noises. Countermeasures against noise pollution could be the choice for the inverted light cycle, rats would be active during working hours when environmental noise is greater as well as a positive conditioning association of 
human voice as described in gentle handling and tickling.

\section{- Odours}

Pheromones mediate sexual attraction, parental care and agonistic confrontation, whereas kairomones of predators elicit defensive behaviours in rats. Threatening stimuli promotes alarm pheromonal communication and rats can be stressed by odours emitted by others rats. In contrast, non-stressed rats release an appeasing olfactory signal which ameliorates stress responses elicited experimentally in odour recipients rats ${ }^{36}$. The interesting fact is that an odour can be itself either a threatening or an appeasing stimulus. Scents released during negative or positive experiences can make those areas aversive or more attractive, respectively inside experimental apparatus, thus influencing results in subsequent rats. This creates a great challenge because cleaning agents are used in experimental apparatus without any validation about their efficacy and even if they have intrinsic odours that could affect rats. Different humans have different odours and new handlers can elicit stress responses. All the staff must be trained to prevent odour contamination because rats will perceive and react to odours undetected (or well tolerated) by humans.

\section{- Bedding}

Researchers should verify whether practices regarding type of bedding and frequency of cage-cleaning adopted as standard by the institution are adequate to their specific research lines, or if any adjustment should be made ${ }^{37}$. The bedding changes are necessary for a hygienic environment. Nonetheless, it must be highlighted that scent is important for rat and excessive cleaning frequency can impair welfare $^{38}$.
Perhaps, for the decision making of a changing beddings programme, it is more relevant to purposely avoid association with important events for the experiment than the frequency itself. Bedding changes are at least a minor stressor through noise, olfactory and physical disturbance of the cage and should be standardized scheduled between experiments.

\section{Temperature and humidity}

The ambient temperature range in which thermoregulation occurs without the need to increase metabolic heat production or activate evaporative heat loss mechanisms is called the thermoneutral zoneand it is bounded by the lower and upper critical temperatures. The U.S. National Research Council (NRC) recommends housing laboratory rat in environmental temperatures ranging from 20 Celsius (68 Fahrenheit) to $26 \circ \mathrm{C}$ (78.8으), despite that the lower critical temperature for the rat was established at $28^{\circ} \mathrm{C}\left(82.4^{\circ} \mathrm{F}\right)^{39}$. It is common to retrieve papers which describes that rats were maintained at standard NRC lower temperatures recommendations than in temperatures equal or superior to $28^{\circ} \mathrm{C}\left(82.4^{\circ} \mathrm{F}\right)$. It is possible that most of the experiments are conducted in temperatures that are comfortable for humans but not for rats. Maximal REM sleep time defined a narrower thermoneutral zone than oxygen consumption. The maximal REM sleep time was at $29^{\circ} \mathrm{C}\left(84.2^{\circ} \mathrm{F}\right)$ and it was more than double the amount seen at $23^{\circ} \mathrm{C}(73.4$ 으), which is a standard laboratory temperature ${ }^{40}$.

The surgery room temperature is extremely important. The range room temperature that is pleasing for humans is too low for the rat which can easily suffer of hypothermia during anaesthesia.

The acceptable range of relative humidity is considered to be $30 \%$ to $70 \%$ for most mammalian species. A narrower band of adequate relative humidity specific for the 
laboratory rat is yet to be determined.

\section{Vocal communication}

Rats produce audible squeaks and at least three types of ultrasonic vocalisations. One type is specific for juvenile rats, pups use it to communicate with their mothers and it is a $40-50 \mathrm{kHz}$ vocalisation. Adult laboratory rats produce two distinct types of ultrasonic vocalization, one associated with positive events and other associated with negative ones. The positive range, commonly named as $50 \mathrm{kHz}$, has a sound frequency ranging from 35 to $70 \mathrm{kHz}$ and it is emitted during and in anticipation of social contact situations, play, sexual behaviour, anticipation of non-social rewarding events and it is also correlated with non-social exploratory behaviour. The ultrasonic vocalisations associated with negative stimuli are known as $22 \mathrm{kHz}$ vocalisations but actually ranges from 18 to $32 \mathrm{kHz}$. They are emitted during, following, or in anticipation of negative events like acute pain, aggressive encounters, during defensive/submissive postures on social defeat and in response to potential predation. Audible squeak occur during playing and fighting and during handling concurrently with struggling. Researchers must be aware that procedures that cause rats to vocalise in order to communicate stress in both sonic and ultrasonic ranges affect the behaviour and physiology of neighbouring rats within audible range, this disturbs welfare and can cause biased results ${ }^{41}$. Rats dislike any kind of physical restraint and usually they will alert others of by sound, ultrasound and odour, so when this is necessary like during preanaesthetic medication, it should be done away of the others. Isolation is stressful for rats, so it should be done only when necessary for the minimal possible time, like during the pre and postoperative period. The researcher will set a standard time that will be used for all subjects of the experiment.

\section{Pain management}

The decisions and reporting of the protocols for intra-operative analgesia, extended postoperative analgesia, and use of multimodal analgesia is not exclusively a welfare issue but an important factor to reproducibility as well and it is an ethical imperative. In 2016, Carbone and Austin reported only $26 \%$ of post-surgical analgesia use in rodents (68 of 261 reviewed papers), and up of $76 \%$ of the scored articles a reader could not know with any certainty whether analgesics were used or not ${ }^{42}$. Analgesia will be employed in clinical studies, so If there is any doubt about how the pain management can interfere with results, this problem should be addressed in the design of the experiment, maybe a pilot experiment with fewer animals just to clarify any doubt or the inclusion of a control group of animals without pain management. Gender differences for the analgesic treatment should be investigated too. The ethic committee on case by case basis will decide if it is acceptable, but for sure, it is a fairish practice to plan, to execute and to report pain management.

\section{- Closing remarks and future perspectives}

Guidelines for transparency of data regarding rat husbandry and housing before and during experimentation are available for scientific community. There are great opportunities for improvement, particularly with greater adherence by authors and some kind of enforcement of guidelines by editors and reviewers.

Improvements in experiment design and statistical analysis are needed too. Randomized block experimental designs have been widely used in agricultural and industrial research for many decades. Usually they are more powerful, have higher external validity, are less subject to bias, and produce more 
reproducible results than the completely randomized designs typically used in research involving laboratory animals. Reproducibility in animal experimentation can be further increased by using blocking factors as time, sex, age and weight. Similar results in blocks separated in time can be an anticipation of the reproducibility of the experiment6. Multi-laboratory experiments are a promising possibility for improvement of reproducibility and data transparency.

Some editors have adopted measures in order to improve quality of reporting like removal of space restrictions on methods sections of submitted papers to allow authors to describe studies comprehensively5. If space limitation requires the exclusion from the primary paper of any information about material and methods, supplementary materials should be available in a public repository. If none data sharing resources could be used, at least a wide world web site with the complementary data should be informed. These secondary resources allow full disclosure of information with no editorial constraints and must include the raw data of the results including null results. Inevitable null results may be valuable for other researchers

\section{References}

1. Collins FS, Tabak LA. Policy: NIH plans to enhance reproducibility. Nature. 2014;505(7485):612-3. PMID: 24482835.

2. Freedman LP, Cockburn IM, Simcoe TS. The economics of reproducibility in preclinical research. PLoS Biol. 2015;13(6):e1002165. PMID: 26057340.

3. Kilkenny C, Parsons N, Kadyszewski E, Festing MFW, Cuthill IC, Fry D, Hutton J, Altman DG. Survey of the quality of experimental design, statistical analysis and reporting of research using animals. PLoS One. 2009; 4(11):e7824. PMID: 19956596.

4. Kilkenny C, Browne W, Cuthill IC, Emerson M, Altman DG. Animal research: reporting in vivo experiments: the ARRIVE guidelines. Br J Pharmacol. 2010;160(7):1577-9. PMID: 20649561.
5. Baker D, Lidster K, Sottomayor A, Amor S. Two years later: journals are not yet enforcing the ARRIVE guidelines on reporting standards for pre-clinical animal studies. PLoS Biol. 2014;12(1):e1001756. PMID: 24409096.

6. Festing MF. Randomized block experimental designs can increase the power and reproducibility of laboratory animal experiments. Ilar J. 2014;55(3):472-6. PMID: 25541548.

7. Irving $A A$, Yoshimi $K$, Hart $M L$, Parker $T$, Clipson L, Ford MR, Kuramoto T, Dove WF, Amos-Landgraf JM. The utility of Apc-mutant rats in modeling human colon cancer. Dis Model Mech. 2014;7(11):1215-25. PMID: 25288683.

8. Pecoraro V, Moja L, Dall'Olmo L, Cappellini G, Garattini S. Most appropriate animal models to study the efficacy of statins: a systematic review. Eur J Clin Invest. 2014;44(9):848-71. PMID: 25066257.

9. Fernandes MR, Lima NV, Rezende KS, Santos IC, Silva IS, Guimaraes RC. Animal models of obesity in rodents. An integrative review. Acta Cir Bras. 2016;31(12):840-4. PMID: 28076509.

10. Sengupta P. The laboratory rat: relating its age with human's. Int J Prev Med. 2013;4(6):62430. PMID: 23930179.

11. Zucker I, Beery AK. Males still dominate animal studies. Nature. 2010;465(7299):690. PMID: 20535186.

12. Tannenbaum C, Schwarz JM, Clayton JA, de Vries GJ, Sullivan C. Evaluating sex as a biological variable in preclinical research: the devil in the details. Biol Sex Differ. 2016;7. PMID: 26870316.

13. Prendergast BJ, Onishi KG, Zucker I. Female mice liberated for inclusion in neuroscience and biomedical research. Neurosci Biobehav Rev. 2014;40:1-5. PMID: 24456941.

14. Clayton JA. Studying both sexes: a guiding principle for biomedicine. FASEB J. 2016;30(2):519-24. PMID: 26514164.

15. Pritchett-Corning KR, Prins JB, Feinstein $R$, Goodwin J, Nicklas W, Riley L. AALAS/FELASA Working Group on Health Monitoring of rodents for animal transfer. J Am Assoc Lab Anim Sci. 2014;53(6):633-40. PMID: 25650968.

16. Bleich A, Hansen AK. Time to include the gut microbiota in the hygienic standardisation of laboratory rodents. Comp Immunol 
Microbiol Infect Dis. 2012;35(2):81-92. PMID: 22257867.

17. Chow down. Scientists should pay more heed to the varying effects of diet and environment on animal work. Nature News. 2016;530(7590):254. PMID: 26887455.

18. Martin B, Ji S, Maudsley S, Mattson MP. "Control" laboratory rodents are metabolically morbid: why it matters. Proc Natl Acad Sci U S A. 2010;107(14):6127-33. PMID: 20194732.

19. Gomez-Smith M, Karthikeyan S, Jeffers MS, Janik R, Thomason LA, Stefanovic B, Corbett D. A physiological characterization of the Cafeteria diet model of metabolic syndrome in the rat. Physiol Behav. 2016;167:382-391. PMID: 27705750

20. Reed DR, Duke FF, Ellis HK, Rosazza MR, Lawler MP, Alarcon LK, Tordoff MG. Body fat distribution and organ weights of 14 common strains and a 22-strain consomic panel of rats. Physiol Behav. 2011;103(5):523-9. PMID: 21504752.

21. Pitts GC. Body composition in the rat: interactions of exercise, age, sex, and diet. Am J Physiol. 1984;246(4 Pt 2):R495-501. PMID: 6720924.

22. Hendershott TR, Cronin ME, Langella $S$, McGuinness PS, Basu AC. Effects of environmental enrichment on anxiety-like behavior, sociability, sensory gating, and spatial learning in male and female C57BL/6J mice. Behav Brain Res. 2016;314:215-25. PMID: 27498148.

23. Kawano T, Eguchi S, Iwata H, Tamura T, Kumagai N, Yokoyama $M$. Impact of preoperative environmental enrichment on prevention of development of cognitive impairment following abdominal surgery in a rat model. Anesthesiology. 2015;123(1):16070. PMID: 26001032.

24. Burke DA, Magnuson DS, Nunn CD, Fentress KG, Wilson ML, Shum-Siu AH, Moore MC, Turner LE, King WW, Onifer SM. Use of environmentally enriched housing for rats with spinal cord injury: the need for standardization. J Am Assoc Lab Anim Sci. 2007;46(2):34-41. PMID: 17343351.

25. Hirsjarvi $P$, Valiaho T. Effects of gentling on open-field behaviour of Wistar rats in fear-evoking test situation. Lab Anim. 1995;29(4):380-4. PMID: 2274759.

26. Cloutier S, Wahl KL, Panksepp J, Newberry,
R.C. Playful handling of laboratory rats is more beneficial when applied before than after routine injections. Appl Anim Beh Sci. 2016;164:81-90. PMID: 24602543.

27. Clough G. Environmental effects on animals used in biomedical research. Biol Rev Camb Philos Soc. 1982;57(Pt 3):487-523. PMID: 6982731.

28. Noell WK, Walker VS, Kang BS, Berman S. Retinal damage by light in rats. Invest Ophthalmol. 1966;5(5):450-73. PMID: 5929286.

29. Beck W, Gobatto C. Effect of high wavelengths low intensity light during dark period on physical exercise performance, biochemical and haematological parameters of swimming rats. Physiol Int. 2016;103(1):112-9. PMID: 27030633.

30. Abou-Ismail UA. The effects of cage enrichment on agonistic behaviour and dominance in male laboratory rats (Rattus norvegicus). Res Vet Sci. 2011;90(2):346-51. PMID: 20594567.

31. Nelson RJ, Moffatt CA, Goldman BD. Reproductive and nonreproductive responsiveness to photoperiod in laboratory rats. J Pineal Res. 1994;17(3):123-31. PMID: 7897584.

32. Francisco NR, Raymond CM, Heideman PD. Short photoperiod inhibition of growth in body mass and reproduction in $\mathrm{ACl}$, BUF, and PVG inbred rats. Reproduction. 2004;128(6):857-62. PMID: 15579603.

33. Heideman PD, Bierl CK, Galvez ME. Inhibition of reproductive maturation and somatic growth of Fischer 344 rats by photoperiods shorter than L14:D10 and by gradually decreasing photoperiod. Biol Reprod. 2000;63(5):1525-30. PMID: 11058561.

34. Wiberg GS, Grice HC. Long-term isolation stress in rats. Science. 1963;142(3591):507. PMID: 14064449.

35. Sharp J, Zammit T, Azar T, Lawson D. Stresslike responses to common procedures in individually and group-housed female rats. Contemp Top Lab Anim Sci. 2003;42(1):9-18. PMID: 12580569.

36. Kiyokawa Y. Social odors: alarm pheromones and social buffering. Curr Top Behav Neurosci. 2017;30:47-65. PMID: 26602247.

37. Horn MJ, Hudson SV, Bostrom LA, Cooper DM. Effects of cage density, sanitation frequency, and bedding type on animal 
wellbeing and health and cage environment in mice and rats. J Am Assoc Lab Anim Sci. 2012;51(6):781-8. PMID: 23294884.

38. Burn CC, Peters A, Day MJ, Mason GJ. Longterm effects of cage-cleaning frequency and bedding type on laboratory rat health, welfare, and handleability: a cross-laboratory study. Lab Anim. 2006;40(4):353-70. PMID: 17018207.

39. Gordon CJ. Thermal biology of the laboratory rat. Physiol Behav. 1990;47(5):963-91. PMID: 2201986.

40. Szymusiak R, Satinoff E. Maximal REM sleep time defines a narrower thermoneutral zone than does minimal metabolic rate. Physiol Behav. 1981;26(4):687-90. PMID: 7267757.

41. Simola N. Rat Ultrasonic Vocalizations and Behavioral Neuropharmacology: From the Screening of Drugs to the Study of Disease. Curr Neuropharmacol. 2015;13(2):164-79. PMCID: PMC4598429.

42. Carbone L, Austin J. Pain and laboratory animals: publication practices for better data reproducibility and better animal welfare. PLoS One. 2016;11(5):e0155001. PMID: 27171143.

\section{Correspondence:}

José Luiz Fontoura-Andrade

Hospital das Forças Armadas

Setor HFA, Sudoeste

70673-900 Brasília - DF Brasil

Tel.: (55 61)3966-2555

divisaodepesquisa@hfa.mil.br

Received: Nov 28, 2016

Review: Jan 26, 2017

Accepted: Feb 24, 2017
Financial source: none

Conflict of interest: none
${ }^{1}$ Research performed at Postgraduate Program in Medical Sciences, School of Medicine, Universidade de Brasília (UnB), and Armed Forces Hospital, Brasilia-DF, Brazil. 